\title{
Is general relativity an "already parametrized" theory?
}

\author{
C. G. Torre \\ Department of Physics, Utah State University, Logan, Utah 84322-4415
}

(Received 26 May 1992)

\begin{abstract}
Beginning with the work of Dirac and of Arnowitt, Deser, and Misner in the late 1950s and early 1960 s, and then after subsequent development by Kuchař, the canonical dynamical structure of general relativity has often been viewed as that of a parametrized field theory in which the many-fingered spacetime variables are hidden among the geometrodynamical field variables. This paradigm of general relativity as an "already parametrized" theory forms the basis for one of the most satisfactory resolutions of the problems of time and observables in classical and quantum gravity. However, despite decades of effort, no identification of many-fingered spacetime variables has ever been satisfactorily obtained for vacuum general relativity. We point out that there is an obstruction to identifying the constraint surface of general relativity (for the case of a closed universe) with that of any parametrized theory. Therefore, strictly speaking, general relativity cannot be viewed as a parametrized field theory. We discuss implications for the canonical quantization program.
\end{abstract}

PACS number(s): 04.20.Cv, 04.60. $+\mathrm{n}$

Beginning in the late 1950s and early 1960s with the work of Dirac [1] and of Arnowitt, Deser, and Misner [2] the canonical dynamical structure of general relativity has often been viewed as that of a "parametrized field theory": a generally covariant version of any field theory obtained by adjoining many-fingered spacetime variables-spacelike embeddings and their conjugate momenta - to the original set of canonical field variables (see, for example, [3]). A decade later Kuchař extensively developed the structure of parametrized field theories and their role as a paradigm for canonical gravity $[4,5]$. Essentially, the idea behind this work is that the embedding variables are somehow camouflaged among the canonical coordinates and momenta of geometrodynamics, and as these variables define (internally specified) spacetime points, their canonical conjugates must necessarily be identified with energy and momentum densities for the true degrees of freedom of the gravitational field. From the point of view of parametrized field theory this identification constitutes the meaning of the Hamiltonian and momentum constraints which appear as a subset of the Einstein equations.

It is not hard to see why this paradigm was so attractive to so many workers in general relativity. Classically, if canonical gravitational dynamics can be cast into the mold of parametrized field theory then one has a clean separation between kinematical (or "pure gauge") aspects of general relativity and the truly dynamical aspects. In particular the Cauchy problem becomes especially simple because the constraints reduce to conditions on the embedding momenta and it is then quite clear what the freely specifiable initial data are.

While the parametrized field-theory paradigm leads to a pleasantly simple picture of classical dynamics of the gravitational field, it is when trying to understand the quantum mechanics of the gravitational field that this paradigm becomes extremely attractive. If the embeddings and their conjugate momenta can be gleaned from the phase space of general relativity then it is straightforward, at least formally, to construct a resolution of the well-known problems of time and observables [6,5]. Briefly stated, the problem of time is concerned with the absence of any structure that can be taken as time for the purposes of quantization. If the many-fingered spacetime variables can be isolated, then one has internally specified "rods and clocks" that can be used for the construction and interpretation of quantum theory. Closely related to the problem of time is the problem of observables, which arises from the fact that not a single observable, i.e., function on the constraint surface that is invariant under the canonical transformations generated by the constraints, is known in general relativity (in the case of a closed universe). This spells difficulty for interpretation of quantum gravity formulated in the manner of Dirac, where one is to select physical states of the gravitational field by demanding they satisfy operator versions of the Hamiltonian and momentum constraints. In this approach to quantization it is only the observables that have a chance to be promoted to (self-adjoint) operators on the physically allowed (Hilbert) space of states. In the quantum version of the parametrized formalism, the constraints become functional Schrödinger equations and it is then consistent to take the observables to be (functions of) the freely specifiable Cauchy data [5]. Wave functions satisfying the functional Schrödinger equations represent probability amplitudes for measuring the free Cauchy data on a hypersurface set by the embedding variables.

Despite decades of effort, it has proved a very difficult task to implement the point of view that general relativity is a parametrized field theory. The best attempt in the context of the vacuum theory is related to the conformal approach to the initial value problem [7] in which the role of many-fingered time is given to the mean extrinsic curvature of the hypersurface upon which the canonical data are defined. However, despite the great utility of this formalism for attacking the Cauchy problem in gen- 
eral relativity, it is not yet adequate for solving the fundamental problems of canonical quantum gravity [5]. If one allows coupling to matter, the situation can improve rather dramatically [8], but even when using matter to give invariant meaning to spacetime points there are still some drawbacks [5].

A good deal of effort has been devoted to the study of simple models that allow for the implementation of the parametrized theory paradigm with varying degrees of success [5], and such investigations expose the difficulties that can occur. But, because one is dealing with models, one is never sure exactly what is going to be the situation in the full theory. The current state of affairs is that very little is known about the possibility of transforming the full vacuum theory into the parametrized formalism.

The purpose of this article is effectively to place an upper bound on the degree to which one can view general relativity as a parametrized field theory, and discuss the implications for canonical quantization based on this paradigm. More precisely, we will point out that there is an obstruction to finding a bijection that would identify the constraint surface of general relativity with that of any parametrized field theory. The proof relies heavily on results and techniques of Arms, Fischer, Isenberg, Marsden, and Moncrief [9] and is essentially a reductio ad absurdum. Throughout we confine our attention to closed universes; i.e., the spacetime manifold is $M=R \times \Sigma$ with $\Sigma$ compact.

Let $(\Gamma, \Omega)$ denote the phase space and symplectic structure for general relativity. $\Gamma$ is taken to be a (suitably defined [9]) cotangent bundle over the space of three-metrics on a compact three-dimensional manifold $\Sigma$ and $\Omega$ is its canonical sympletic structure. Dynamically admissible points in $\Gamma$ lie on the constraint surface $\bar{\Gamma} \subset \Gamma$, which satisfies the Hamiltonian and momentum constraints:

$$
H_{\perp}=0=H_{a} \text {. }
$$

Let $(\Upsilon, \omega)$ denote the phase space of a parametrized field theory and its symplectic structure. $\Upsilon$ is the product of the cotangent bundle over embeddings of $\Sigma$ into the spacetime manifold $\boldsymbol{M}$ (equipped with the canonical symplectic structure) and the phase space for a field theory, which is taken to be an infinite-dimensional symplectic manifold. Denote points in the phase space for the field theory as $Z^{A}$, which are a collection of fields on $\Sigma$. Let $Q: \Sigma \rightarrow M$ represent an embedding, and denote the momentum conjugate to the embedding as $P_{\alpha}$, which represents a cotangent vector to the space of embeddings. Dynamically allowed points in $\Upsilon$ also lie on a constraint surface $\bar{\Upsilon} \subset \Upsilon$ defined by

$$
\Pi_{\alpha}:=P_{\alpha}+h_{\alpha}\left(Q, Z^{A}\right)=0
$$

Here $h_{\alpha}$ has the physical interpretation as the flux of energy-momentum of the fields $Z^{A}$ through the hypersurface defined by $Q$.

We can now make precise the sense in which one would like to view general relativity as a parametrized field theory.

Conjecture. There is a bijection $\Phi: \Upsilon \rightarrow \Gamma$ which identifies the constraint surface of general relativity with that of a parametrized field theory:

$$
\Phi(\bar{\Upsilon})=\bar{\Gamma} \text {. }
$$

The conjecture amounts to the statement that there is a change of variables on the phase space of general relativity such that, using the Hamiltonian and momentum constraints, $4 \infty^{3}$ of the new variables can be solved for in terms of the remaining variables. In practice one would like to make the conjecture a good bit stronger. First, one would like to demand the map $\Phi$ is a diffeomorphism; further, this diffeomorphism should identify the symplectic structures on $\Gamma$ and $\Upsilon$, i.e., $\omega=\Phi^{*} \Omega$. This means that the change of variables mentioned above is in fact a canonical transformation (this requirement can be weakened somewhat). Second, it is necessary that the embeddings $Q$ are spacelike with respect to the Einstein metrics which solve the Hamiltonian equations of motion. These addenda to the above conjecture are irrelevant for our present purposes because the conjecture already fails without the added assumptions.

The conjecture fails because $\bar{\Gamma}$ is a stratified manifold, i.e., it has "conical singularities" at phase space points representing Cauchy data for spacetimes with Killing vectors [9]. It is precisely these singular points which prevent one from solving the constraints as indicated by the conjecture. More precisely, it can be shown that $\bar{\Upsilon}$ is everywhere a smooth manifold, and hence there cannot be a bijection that identifies $\bar{\Upsilon}$ and $\bar{\Gamma}$. The conjecture is false.

The proof that $\bar{\Upsilon}$ is globally a manifold follows a model calculation of Arms [10]. Let $\mathscr{E}$ represent the space of cotangent vectors to the space of embeddings. Define the map $\Pi: \Upsilon \rightarrow \mathscr{E}$ via $\bar{\Upsilon}=\Pi^{-1}(0)$. If the differential $d \Pi$, which is a linear map from the tangent space at a point of $\Upsilon$ to $\mathscr{E}$ obtained by linearizing the constraints, is surjective at a point in $\bar{\Upsilon}$, then, using the implicit function theorem [11], there is a neighborhood $U \subset \bar{\Upsilon}$ of that point which is a smooth submanifold of $\Upsilon$. Surjectivity follows if it can be shown that the natural adjoint of $d$ II is injective and has injective symbol everywhere on $\bar{\Upsilon}$ [9]. The injective symbol implies that the adjoint operator $d \Pi^{*}$ is elliptic so that there is an orthogonal decomposition

$$
\mathscr{E}=\operatorname{Range}(d \Pi) \oplus \operatorname{Kernel}\left(d \Pi^{*}\right) .
$$

If $d \Pi^{*}$ is itself injective, i.e., $\operatorname{Kernel}\left(d \Pi^{*}\right)=0$, then from the above equation $d \Pi$ is surjective. It is straightforward to verify that at each point of $\bar{\Upsilon}$ the symbol of the adjoint operator is injective (in the generalized sense of [12]) as is the adjoint operator itself, and hence the map $\frac{d}{\Upsilon} \Pi$ is surjective everywhere on $\bar{\Upsilon}$. This guarantees that $\bar{\Upsilon}$ is everywhere a manifold.

The essence of the difficulty with the conjecture seems to be that it is impossible for the first and second fundamental forms (metric and extrinsic curvature-canonical coordinates and momenta) of a hypersurface to uniquely determine the embedding $Q$ of that hypersurface into a spacetime with symmetry. Indeed, it can be shown that if a spacetime possesses a Killing vector, then typically there is a one-parameter family of embeddings corre- 
sponding to each choice of first and second fundamental forms (satisfying the Gauss-Codazzi equations).

So it seems that the best one can hope for in general relativity is to find an identification of gravitation as a parametrized field theory only for the generic spacetimes, i.e., those without symmetry. Note that, in particular, this means there can never be a completely successful "deparametrization" of Einstein's theory of gravitation. To put this result into a proper perspective we must see (i) what assumptions in the proof can be sensibly relaxed, and (ii) what it implies in practice, e.g., for canonical quantum gravity. While such an investigation is obviously beyond the possible scope of this article, we can make a few comments here.

It is tempting to think that one should relax the assumption that the phase space of fields $Z^{A}$ is a (symplectic) manifold because this would allow for the possibility that both $\bar{\Upsilon}$ and $\bar{\Gamma}$ are manifolds with singularities. The problem with this strategy is that, because $\Gamma$ is a manifold, it is now impossible to find a diffeomorphism that identifies $\Gamma$ and $\Upsilon$, and so this obvious modification of the conjecture is still problematic.

A more intriguing way to get around the obstruction encountered above is to redefine the gravitational phase space, a prime example of this being Ashtekar's Hamiltonian formulation of general relativity [13]. Here one can attempt to identify the constraint surface of general relativity with that of a parametrized gauge theory. Because the constraint surface for a gauge theory (even without parametrization) is not necessarily a manifold [14], the argument given above does not need to apply. The formulation of parametrized gauge theory in general and particularly its use as a paradigm for Ashtekar's formulation of general relativity are topics worthy of further investigation.

In the spirit of trying to find a phase space formulation of general relativity that is more amenable to the parametrized field-theory paradigm, it is worth pointing out that the "covariant phase space" approach to gravitation [15] is available. In this picture the putative relation with parametrized field theory may change considerably. Indeed, the covariant phase space formulation of parametrized field theory is substantially different from its canonical counterpart under consideration here [16]. This is another topic worthy of further investigation.

Returning back to the implications of the obstruction for the traditional canonical formulation of general relativity, there is a potential mitigating factor that is worth pointing out. It is possible that "most" spacetime manifolds simply do not admit Killing vectors because the manifold structure of $\Sigma$ is too wild. It is hard to assess the impact of this observation for canonical gravity for two reasons. First of all, it is not yet known precisely "how many" three-manifolds would or would not allow for Killing vectors. Second, in the canonical formulation of Einstein's theory of gravitation the spatial manifold $\Sigma$ must be specified externally and is not really a variable in the theory; it is hard to decide how important a given spatial manifold should be from purely theoretical considerations.

Let us conclude by discussing the quantum mechanical implications of the obstruction to the above conjecture. Here we must, at present, resort to models of quantum gravity. Two (very similar) models can be exhibited which nicely illustrate the type of obstruction we are discussing. One is a model of Kuchar $[17,10,18,19]$, the techniques of which are easily generalized [20,21] to the "midi-superspace" of spacetimes that admit two commuting Killing vectors (compact spatial topology in both cases). These are the so-called "Gowdy models." To be concrete, let us focus on the case where the universe has the topology of a three-torus. It is possible to extend the results of [20] to this case and it can be shown [20] that there is a map which identifies the constraint surface of the Gowdy spacetimes with that of a parametrized field theory (essentially a nonlinear $\sigma$ model), i.e., a map that satisfies (1), but this map is not bijective. Alternatively, one can find a bijection between the gravitational model ${ }^{1}$ and the model parametrized theory, but the gravitational constraint surface appears as a submanifold (with singularities) of the constraint surface of the parametrized field theory [so that (1) is violated]. As shown in [19], in the quantum theory this latter possibility leads to a set of functional Schrödinger equations, and all the advantages associated with such equations, along with a finite number of subsidiary conditions to be placed on the quantum mechanical state vector. Therefore, in these models, the physical interpretation allowed by the parametrized field-theoretic structure remains intact, but the notion of observable has to be modified slightly to be consistent with the subsidiary conditions. If this situation could be shown to persist in the full theory, the failure of the conjecture would not necessarily preclude the use of the paradigm to resolve the difficult conceptual and technical issues facing canonical quantum gravity.

I would like to thank Abhay Ashtekar and Karel Kuchar for discussions.

\footnotetext{
${ }^{1}$ Actually, in this case one embeds the model gravitational phase space in a (slightly) larger phase space, much as we suggested might be useful using, e.g., the Ashtekar formalism.
}

[1] P. A. M. Dirac, Lectures on Quantum Mechanics (Yeshiva University, New York, 1964).

[2] R. Arnowitt, S. Deser, and C. Misner, in Gravitation: An Introduction to Current Research, edited by L. Witten (Wiley, New York, 1962).

[3] A. Hanson, T. Regge, and C. Teitelboim, Constrained Hamiltonian Systems (Accademia Nazionale dei Lincei, Rome, 1976).
[4] K. V. Kuchař, J. Math. Phys. 13, 758 (1972); 17, 801 (1976).

[5] K. V. Kuchař, in Proceedings of the Fourth Canadian Conference on General Relativity and Relativistic Astrophysics, edited by G. Kunstatter, D. Vincent, and J. Williams (World Scientific, Singapore, 1992).

[6] See Conceptual Problems of Quantum Gravity, edited by A. Ashtekar and J. Stachel (Birkhäuser, Boston, 1991). 
[7] See, e.g., Y. Choquet-Bruhat and J. York, in General Relativity and Gravitation: 100 Years After the Birth of Albert Einstein, edited by A. Held (Plenum, New York, 1980), Vol. 1.

[8] K. V. Kuchař and C. G. Torre, Phys. Rev. D 43, 419 (1990); 44, 3116 (1991).

[9] See A. Fischer and J. Marsden, in General Relativity: An Einstein Centenary Survey, edited by S. Hawking and W. Israel (Cambridge University Press, Cambridge, England, 1979); J. Isenberg and J. Marsden, Phys. Rep. 89, 181 (1982), and references therein.

[10] J. Arms, J. Math. Phys. 21, 15 (1980).

[11] Y. Choquet-Bruhat, C. DeWitt-Morette, and M. DillardBleick, Analysis, Manifolds and Physics (North-Holland, New York, 1982).

[12] L. Hörmander, Ann. Math. 83, 129 (1966).

[13] See A. Ashtekar, Lectures on Non-Perturbative Canonical Gravity (World Scientific, Singapore, 1991), and references therein.
[14] J. Arms, J. Math. Phys. 20, 443 (1979).

[15] C. Crnkovic and E. Witten, in 300 Years of Gravitation, edited by $\mathrm{S}$. Hawking and $\mathrm{W}$. Israel (Cambridge University Press, Cambridge, England, 1987); J. Lee and R. Wald, J. Math. Phys. 31, 725 (1990); A. Ashtekar, L. Bombelli, and O. Reula, in Mechanics, Analysis and Geometry: 200 Years After Lagrange, edited by M. Francaviglia (NorthHolland, New York, 1991).

[16] C. G. Torre, J. Math. Phys. (to be published).

[17] K. V. Kuchař, J. Math. Phys. 19, 390 (1978).

[18] K. V. Kuchař and C. G. Torre, J. Math. Phys. 30, 1769 (1989).

[19] K. V. Kuchař and C. G. Torre, in Conceptual Problems of Quantum Gravity [6].

[20] C. G. Torre (unpublished).

[21] For the case in which the Killing vectors are hypersurface orthogonal and the universe is open, see K. V. Kuchar, Phys. Rev. D 4, 955 (1971); C. G. Torre, Class. Quantum Grav. 8, 1895 (1991). 